\title{
Evaluation of aortic intima-media thickness in newborns with Down syndrome
}

\author{
Dilek Sarici, A-F, Selim Kurtoglu ${ }^{2, A, E, F}$, Serdar Umit Saricici, C-F , Ali Yikilmaz $z^{4, B}$, Mustafa Ali Akinn 2, B, Tamer Gunes 2, A, E, F, \\ Mehmet Adnan Ozturk ${ }^{2, A, E, F}$, Nazmi Narin ${ }^{5, B, F}$, Munis Dündar ${ }^{6, B}$, Muhittin Serdar, ${ }^{7, C}$ \\ ${ }^{1}$ Division of Neonatology, Department of Pediatrics, Keçiören Training and Research Hospital, Ankara, Turkey \\ ${ }^{2}$ Division of Neonatology, Department of Pediatrics, Faculty of Medicine, Erciyes University, Kayseri, Turkey \\ ${ }^{3}$ Division of Neonatology, Department of Pediatrics, Faculty of Medicine, Ufuk University, Ankara, Turkey \\ ${ }^{4}$ Department of Radiology, Faculty of Medicine, Erciyes University, Kayseri, Turkey \\ ${ }^{5}$ Division of Cardiology, Department of Pediatrics, Faculty of Medicine, Erciyes University, Kayseri, Turkey \\ ${ }^{6}$ Department of Medical Genetics, Faculty of Medicine, Erciyes University, Kayseri, Turkey \\ ${ }^{7}$ Department of Biochemistry, Faculty of Medicine, Acıbadem University, Istanbul, Turkey \\ A - research concept and design; $\mathrm{B}$ - collection and/or assembly of data; $\mathrm{C}$ - data analysis and interpretation; \\ $D$ - writing the article; $E$ - critical revision of the article; $F$ - final approval of the article
}

Address for correspondence

Dilek Sarici

E-mail:drdilekcoban@yahoo.com.tr

\section{Funding sources}

None declared

Conflict of interest

None declared

Received on July 14, 2015

Reviewed on September 7, 2016

Accepted on January 26, 2017

DOI

\begin{abstract}
Background. Health care programs for children with Down syndrome (DS) help improve the overall outcome and quality of life of these children. It is therefore very important to focus on the most common and serious problems of these patients, such as congenital heart defects and cardiac problems, and to keep medical guidelines updated with regard to these problems.
\end{abstract}

Objectives. The aim of this study was to evaluate aortic intima-media thickness (aIMT), lipid profiles and blood pressure in DS patients in comparison with a control group of age- and gender-matched neonates without DS.

Material and methods. Serum concentrations of lipids (total cholesterol, LDL-cholesterol, HDL-cholesterol, and triglyceride), blood pressure and abdominal alMT were measured in newborns with DS, and compared with the measurements from age- and gender-matched newborns without DS.

Results. No statistically significant differences between the 2 groups of newborns were detected with respect to alMT, lipid levels or blood pressure.

Conclusions. This study represents the first investigation of alMT - one of the most important indicators of atherosclerosis - in DS patients, but neither a significantly increased aIMT, nor any significant changes in lipid profiles or blood pressure were detected in this group of patients. Whether alMT differs according to the type of congenital heart defect (such as atrial septal defect, ventricular septal defect, patent ductus arteriosus, and atrioventricular septal defect) among DS patients remains to be determined in future studies.

Key words: newborn, Down syndrome, intima-media thickness

\subsection{9/acem/68677}

\section{Copyright}

Copyright by Author(s)

This is an article distributed under the terms of the

Creative Commons Attribution Non-Commercial License

(http://creativecommons.org/licenses/by-nc-nd/4.0/) 
Down syndrome (DS, also known as trisomy 21) is the most common chromosomal abnormality, occurring in approx. 1 in 800 live births. Overexpression of the genes located on chromosome 21 is thought to underlie the pathogenesis of the neurologic, immunologic, endocrine and biochemical abnormalities that are characteristic of the syndrome. Children with DS often have congenital heart defects (CHD), gastrointestinal anomalies, immunologic disorders, and thyroid dysfunction. ${ }^{1,2}$

Approximately $44-58 \%$ of newborns with DS have CHD; atrioventricular septal defect (AVSD) and ventricular septal defect (VSD) are the most commonly encountered forms of CHD. ${ }^{3,4}$ Early recognition of CHD can lead to the optimal management of the defect. The survival of cases with DS has risen from 25 years to 49 years in the USA in a 14-year period, and congenital heart defects and respiratory infections are the most frequently reported causes of death in individuals with DS. ${ }^{5}$ Although modern medical care has reduced the mortality rate to more acceptable values, it is still possible to further reduce both morbidity and mortality. Health care programs for children with DS help improve the overall outcome and quality of life of these children. It is therefore very important to focus on the most common and serious problems for these patients, such as congenital heart defects and cardiac problems, and to keep medical guidelines updated about these complications. ${ }^{6}$

The aim of this study was to evaluate aortic intimamedia thickness (aIMT), lipid profiles and blood pressure in DS patients in comparison with a control group of ageand gender-matched neonates without DS.

\section{Material and methods}

Serum concentrations of lipids (total cholesterol - TC, LDL-cholesterol - LDL-C, HDL-cholesterol - HDL-C and triglyceride - TG), blood pressure, and abdominal aIMT were measured in patients with DS, and compared with those from a control group of age- and gendermatched neonates without DS. All the neonates with DS that were included in the study were born at or referred to the Department of Pediatrics at the Erciyes University, Faculty of Medicine (Kayseri, Turkey) between September 2009 and June 2011. All of them were full-term ( $\geq 38$ gestational weeks). The control group was comprised of newborns who were referred to the well-baby clinic of the Erciyes University Medical Faculty hospital during the same period, and who had no cardiac pathology. The study was approved by the local Ethics Committee, and all the mothers provided written informed consent before enrollment.

Plasma lipid levels were measured in the venous blood of 20 infants with DS (13 males and 7 females) and 20 newborns without DS (also 13 males and 7 females). The blood samples obtained were immediately trans- ferred into glass tubes, separated by centrifugation at $3500 \mathrm{rpm}$ for $4 \mathrm{~min}$, then kept at $-80^{\circ} \mathrm{C}$ until analysis. Serum concentrations of TC, HDL-C, LDL-C and TG were measured by the photometric method adapted to an autoanalyzer (Architect ${ }^{\circledR}$ C16000, Abbott Diagnostics, Lake Forest, USA). LDL-C was directly measured since not all the blood samples could be achieved during fasting.

Infant anthropometry was obtained by a trained research midwife. An infant electronic scale was used to obtain the subjects' weight in grams. The systolic, diastolic and mean blood pressure of the neonates was measured by the oscillometric technique, using a standardized protocol. The infants were studied at least $1.5 \mathrm{~h}$ after the last feeding. An appropriately sized cuff was placed on the right upper arm, and the baby was not touched for at least 15 min or until the subject attained a sleepy or quiet waking state. Three successive measurements were made at 2-min intervals, and the mean value of each measurement (systolic, diastolic and mean blood pressure) was recorded.

For the ultrasound imaging to evaluate aIMT, the subjects were laid quietly in a dark room. The abdominal aortic artery was scanned using a $1200 \times$ Plus high-resolution ultrasound mainframe with 2 linear array probes at 7.5 and $13 \mathrm{MHz}$ (Shimadzu Corp., Kyoto, Japan). Abdominal IMT, defined as the distance between the leading edges of the media-adventitia interface and the blood-intima interface, was measured at a non-branched straight longitudinal part of the distal abdominal aorta, at least $1 \mathrm{~cm}$ long. The measurements were made with a manual measuring system. The dorsal arterial wall of the most distal abdominal aorta was chosen as the measurement site, since this area has been reported as the most lesion-prone. ${ }^{7,8}$ The abdominal aorta was identified in the lower abdomen with a 7.5-MHz linear array transducer, and the aortic intimamedia complex was assessed using a 13-MHz linear array transducer. The same experienced vascular sonographers, who were unaware of the clinical and laboratory situation of the newborns, performed all the ultrasonographic measurements.

All the statistical calculations were performed using SPSS software (v. 15.0, SPSS Inc., Chicago, USA). For continuous variables, the results are presented as the mean \pm SD. Categorical variables are presented as frequency counts. The Shapiro-Wilk test was used to determine the normality of the distribution of the data. The $X^{2}$ test was used to determine whether there was a statistically significant difference between the groups with respect to gender, delivery route and the presence or absence of consanguinity. The independent sample t-test was used to statistically compare demographic parameters (maternal and paternal age, age on admission, gestational age and birth weight), laboratory parameters (aIMT and lipid profiles) and blood pressure parameters between the 2 groups. A p-value $<0.05$ was considered to be statistically significant for all the statistical tests. 


\section{Results}

A comparison of the demographic characteristics of the study and control groups is shown in Table 1 . There were no statistically significant differences between the study and control groups with respect to gender, mode of delivery, consanguinity, maternal and paternal age, age of admission, gestational age or birth weight.

In the study group, there were 3 cases with ASD, 6 cases with VSD, 3 cases with AVSD, and 3 cases with PDA, whereas there were no cases of any cardiac pathology in the control group.

A comparison of the biochemical parameters, aIMT and blood pressure measurements is shown in Table 2 . There were no statistically significant differences between the 2 groups with respect to aIMT, plasma lipid levels or blood pressure measurements.

\section{Discussion}

New studies aimed at improving the management of the heart diseases most commonly affecting mortality and morbidity in patients with DS are needed. To the present

Table 1. Comparison of the demographic characteristics of the study and control groups

\begin{tabular}{|l|c|c|c|}
\hline \multicolumn{1}{|c|}{ Demographic parameters } & $\begin{array}{c}\text { Study group } \\
(\mathbf{n}=\mathbf{2 0})\end{array}$ & $\begin{array}{c}\text { Control group } \\
(\mathbf{n}=20)\end{array}$ & p-value \\
\hline Gender (female/male) & $7 / 13$ & $7 / 13$ & 1.0 \\
\hline Delivery (cesarean section/vaginal) & $16 / 4$ & $13 / 7$ & 0.48 \\
\hline Consanguinity (positive/negative) & $1 / 19$ & $1 / 19$ & 1.0 \\
\hline Maternal age (years) & $31.7 \pm 6.937$ & $28.7 \pm 5.723$ & 0.144 \\
\hline Paternal age (years)* & $35.6 \pm 7.472$ & $32.25 \pm 6.206$ & 0.131 \\
\hline Age on admission (days)* & $10.15 \pm 9.343$ & $10.25 \pm 8.84$ & 0.786 \\
\hline Gestational age (weeks)* & $37.7 \pm 1.26$ & $38.25 \pm 1.71$ & 0.255 \\
\hline Birth weight (g) & $3075 \pm 508$ & $3024 \pm 450$ & 0.741 \\
\hline
\end{tabular}

* - values are given as mean $\pm \mathrm{SD}$.

Table 2. Comparison of alMT measurements, plasma lipid levels and blood pressure measurements of the 2 groups

\begin{tabular}{|l|c|c|c|}
\multicolumn{1}{|c|}{ Parameters } & $\begin{array}{c}\text { Study group } \\
(\mathbf{n = 2 0 )}\end{array}$ & $\begin{array}{c}\text { Control group } \\
(\mathbf{n}=20)\end{array}$ & p-value \\
\hline alMT (mm) & $0.350 \pm 0.04$ & $0.355 \pm 0.04$ & 0.681 \\
\hline Triglyceride level (mg/dL) & $86.4 \pm 25.54$ & $91.2 \pm 58.98$ & 0.740 \\
\hline Total cholesterol level (mg/dL) & $99.4 \pm 18.95$ & $98.25 \pm 33.97$ & 0.896 \\
\hline HDL level (mg/dL) & $31.00 \pm 8.99$ & $29.11 \pm 12.70$ & 0.589 \\
\hline LDL level (mg/dL) & $48.65 \pm 18.65$ & $50.96 \pm 24.78$ & 0.741 \\
\hline Systolic blood pressure $(\mathrm{mm} \mathrm{Hg})$ & $63.60 \pm 9.92$ & $62.6 \pm 9.85$ & 0.751 \\
\hline Diastolic blood pressure $(\mathrm{mm} \mathrm{Hg})$ & $34.75 \pm 6.55$ & $34.75 \pm 12.14$ & 1.000 \\
\hline Mean blood pressure $(\mathrm{mm} \mathrm{Hg})$ & $43.75 \pm 6.27$ & $43.15 \pm 10.24$ & 0.825 \\
\hline
\end{tabular}

Values are given as mean \pm SD. authors' knowledge, there have been no studies investigating aIMT measurements in newborns and children with DS, and only 1 study of carotid intima-media thickness (IMT) in adult patients with DS. ${ }^{9}$ In that study, Draheim et al. assessed the IMT in the left common carotid arteries of 52 adults with DS and in age-, gender- and race-matched adults without DS (mean age $42 \pm 5$ years). Total body fat, blood pressure, fasting lipid profiles, insulin, glucose, C-reactive protein, homocysteine, physical activityand dietary intake were also evaluated. The adults with DS had lower IMT, lower systolic and diastolic blood pressure, and higher C-reactive protein, triglycerides and total body fat in comparison to the controls. Male gender and physical activity were reported as predictors of IMT for adults with DS; fasting insulin, fruit and vegetable intake, lowdensity lipoprotein cholesterol, age, smoking, and gender were predictors of IMT for the controls. The researchers concluded that adults with DS may be protected against atherosclerosis despite elevated total body fat and elevated cardiovascular disease risk factors. ${ }^{9}$ The potential protective mechanisms against atherosclerosis in patients with DS are not clear and need to be investigated. Draheim et al. speculated that patients with DS might have genetic factors that protect them against atherogenesis. Additional studies are needed to demonstrate what genes, if any, are responsible for DS and atherogenesis. There were some limitations in the study by Draheim et al. The study included a limited number of cases with DS (only 52); however, it represents the largest sample of atherosclerotic measurements of patients with DS in the available literature. In the present study, aIMT measurements, plasma lipid levels and blood pressure measurements of the newborns with DS were not significantly different from those of the control group.

There are very few studies of post-mortem examinations of DS patients in relation to atherosclerosis. Post-mortem examination of 5 institutionalized patients with DS (aged 40-66 years) showed no atheroma throughout their cardiovascular systems. The coronary arteries had no atheroma macroscopically, and microscopically showed only slight, diffuse intimal thickening that was normal for the subjects' age. In 2 cases a few fatty streaks were detected at the origin of the main aortic branches. ${ }^{10}$ Moss et al. showed a normal prevalence of pre-atherosclerotic lesions in autopsy examinations of 7 non-institutionalized DS patients (10-25 years); atherosclerotic plaques were observed in 1 of these subjects (aged 14 years) but atheromatous aortic lesions were not seen in the $8^{\text {th }}$ subject (aged 50 years). ${ }^{11}$ In a Finnish post-mortem study, Ylä-Herttuala et al. found a lower percentage of raised lesions and less calcium in the coronary arteries of 15 patients with DS (mean age: 51 years) compared with 6 intellectually disabled and 20 normal controls. Thus, even though the coronary arteries of DS patients were not completely free of 
atherosclerosis, it was milder than in other patients and free-living subjects of the same age. ${ }^{12}$

Several variables may explain the decreased incidence of atherosclerosis in DS. Affected individuals are often institutionalized, and may be exposed to a diet low in animal fats; smoking is also less prevalent than in the general population. Furthermore, some studies have documented significantly lower values of arterial blood pressure than in inmates with other intellectual disabilities. ${ }^{10,13}$ Conflicting reports have been published on DS patients' lipid and lipoprotein concentrations, which are the best documented variables associated with the development of atherosclerosis. Most studies have compared subjects with DS with matched individuals admitted to the same institution for other disabling disorders or with unselected normal controls. Simon et al. found high serum cholesterol levels in young DS patients, whereas subsequent studies did not confirm this observation in groups of affected individuals whose ages ranged from early childhood to the early sixties. ${ }^{10,13-17}$ Triglyceride concentrations have been reported to be decreased, increased or unchanged in patients with trisomy 21 compared with matched controls. ${ }^{10,13,16,17}$ Therefore, in spite of the presence of some classical biochemical risk factors for atherosclerosis, its clinical manifestation is low in DS. The reasons for this remain unclear. In the present study, the plasma triglyceride and cholesterol levels of the patients with DS were not significantly different from those of the control group, and this finding was in accordance with most of the studies in this field.

The most important limitation of the present study was that the limited number of DS patients with a congenital heart disease precluded a comparison of aIMT among the subcategories of DS patients with respect to the type of congenital heart defect.

In the present study, aIMT - one of the most important indicators of atherosclerosis - was investigated in DS patients for the first time, but neither a significantly increased aIMT nor any significant changes in lipid profiles or blood pressure were detected in this group of patients. Whether aIMT differs according to the type of congenital heart defect (ASD, VSD, PDA, and AVSD) among DS patients remains to be determined in future studies.

\section{References}

1. Jannson U, Johansson C. Down syndrome and celiac disease. J Pediatr Gastroenterol Nutr. 1995;21:443-445.

2. Sarici D, Akin MA, Kurtoglu S, Gunes T, Ozturk MA, Akcakus M. Thyroid functions of neonates with Down syndrome. Ital J Pediatr. 2012;38:44.

3. Vis JC, Duffels MG, Winter MM, et al. Down syndrome: A cardiovascular perspective. J Intellect Disabil Res. 2009;53:419-425.

4. Weijerman ME, van Furth AM, Vonk Noordegraaf A, van Wouwe JP, Broers CJ, Gemke RJ. Prevalence, neonatal characteristics and first-year mortality of Down syndrome: A national study. J Pediatr. 2009;152:15-19.

5. Yang $Q$, Rasmussen SA, Friedman JM. Mortality associated with Down's syndrome in the USA from 1983 to 1997: A populationbased study. Lancet. 2002;359:1019-1025.
6. Roizen NJ, Patterson D. Down's syndrome. Lancet. 2003;361: 1281-1289.

7. McGill HC Jr, McMahan CA, Herderick EE, et al. Effects of coronary heart disease risk factors on atherosclerosis of selected regions of the aorta and right coronary artery. PDAY Research Group. Pathobiological determinants of atherosclerosis in youth. Arterioscler Thromb Vasc Biol. 2000;20:836-845.

8. Koklu E, Ozturk MA, Kurtoglu S, Akcakus M, Yikilmaz A, Gunes T. Aortic intima-media thickness, serum IGF-I, IGFBP-3, and leptin levels in intrauterine growth-restricted newborns of healthy mothers. Pediatr Res. 2007;62:704-709.

9. Draheim CC, Geijer JR, Dengel DR. Comparison of intima-media thickness of the carotid artery and cardiovascular disease risk factors in adults with versus without the Down syndrome. Am J Cardiol. 2010;106:1512-1516.

10. Murdoch JC, Rodger JC, Rao SS, Fletcher CD, Dunnigan MG. Down's syndrome: An atheroma-free model? BMJ. 1977;2:226-228.

11. Moss TJ, Austin GE. Pre-atherosclerotic lesions in Down syndrome. J Ment Defic Res. 1980;24:137-141.

12. Yla-Hertmala S, Luoma J, Nikkari T, Kivimaki T. Down's syndrome and atherosclerosis. Atherosclerosis. 1989;6:269-272.

13. Salo MK, Solakivi-Jaakkola T, Kivimaki T, Nikkari T. Plasma lipids and lipoproteins in Down's syndrome. Scand J Clin Invest. 1979;39: 485-490.

14. Simon A, Ludwig CH, Gofman JW, Crook GH. Metabolic studies in mongolism: Serum protein-bound iodine, cholesterol, and lipoprotein. Am J Psychiatry. 1954;111:144-145.

15. Dalderup LM, Affourtit-Leeuw H, Van der Spek PAF, Keller GHM, Duin HJ, De longh $\mathrm{H}$. Serum uric acid and cholesterol levels in mongoloid and nonmongoloid oligophrenics. Br J Psychiatry. 1966;112:91-94.

16. Nishida Y, Akaoka I, Nishizawa T, Maruki M, Maruki K. Hyperlipidaemia in patients with Down's syndrome. Atherosclerosis. 1977;26:369-372.

17. Dorner T, Gaethke AS, Tolksdorf M, Schumann KP, Gustmann H. Cholesterol fractions and triglycerides in children and adults with Down's syndrome. Clin Chim Acta. 1984;42: 307-311. 